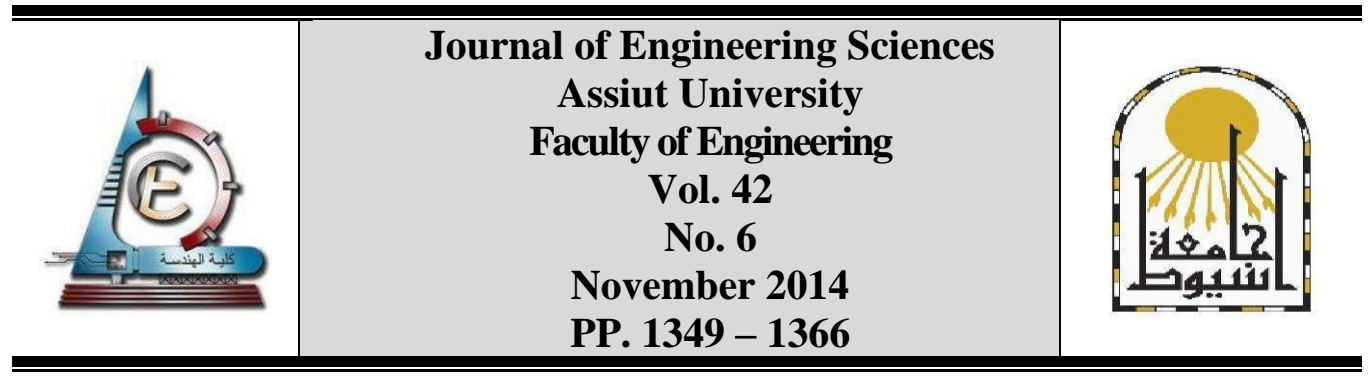

\title{
INVESTIGATION OF AGGREGATE PARTICLES SHAPE ON CHARACTERISTICS OF HOT MIX ASPHALT
}

\author{
Hassan Youness Ahmed ${ }^{1}$, Mostafa Deep Hashem ${ }^{2}$, \\ Naglaa Kamal Rashwan ${ }^{3}$ and Samir Azmy Abdalla ${ }^{4, *}$ \\ ${ }^{1}$ Faculty of Engineering, Assiut University, Civil Engineering Department, EGYPT \\ ${ }^{2}$ Faculty of Engineering, Minia University, Civil Engineering Department, EGYPT \\ ${ }^{3}$ Civil Eng. Dept., Industrial Education College, Beni-Suef, EGYPT \\ ${ }^{4}$ Civil Engineering Dept., Higher Institute of Engineering and Technology, El-Minia, Egypt.
}

(Received 25 November 2014; Accepted 25 December 2014)

\begin{abstract}
This research represents a laboratory study of the effect of the aggregate shape in HMA. The shape of aggregates particles is not the only factor of aggregate characteristics which effect on HMA. Other Parameters such as aggregate gradation and aggregate surface texture are also effect. The aggregate shapes considered for the study were cubical, disk and blade shapes. Two aggregate surface textures used in the mix which were smooth and rough particles. The aggregate gradations used in the mixtures were maximum, medium and minimum gradation. Shape of aggregate was determined through two methods. The first method was an image measurement of coarse aggregate while the second method was particle shape index. Marshal tests and indirect tensile test were performed to investigate the different in behaviors of different samples with different parameters considered in this study taking into account that the control mix contains crushed gravel, rough aggregate particles with disk shape, medium gradation of aggregate and $4 \%$ of limestone powder as mineral filler.

The results showed that using of cubical aggregate shape increased each of Marshall Stability, bulk density, Marshall Stiffness, Marshall Quotient, Stiffness Modulus and the Indirect Tensile Strength of the mix than disk and blade shapes while decreased each of flow and air voids. The results also, showed that medium gradation of aggregate had good results than other gradations. By using rough particles the values of ITS and stiffness modulus were higher than using smooth particles.
\end{abstract}

Keyword: HMA, Aggregate, Aggregate shape, Gradation and Mineral filler.

\section{Introduction}

The mineral aggregate, including coarse and fine particles in asphalt paving mixtures, encompasses approximately $90 \%$ of HMA by volume. The properties of the aggregate have direct and significant effects on the performance of asphalt pavements. Research has shown that aggregate characteristics such as particle size, shape and texture influence the

* Corresponding author.

Email address: civileng.semsem@yahoo.com 
performance and service ability of hot-mix asphalt pavement [1,2,3]. Many studies emphasized the role of aggregate properties in controlling the performance of asphalt mixtures. For example, Kandhal and Parker pointed out that the presence of excessive flat and elongated aggregate particles is undesirable in HMA mixtures because such particles tend to break down during production and construction, thus affecting the durability of HMA mixtures [4]. Cheng and Chang found that Cubical particles possessed the best rutting resistance over the other shapes. Flaky and/or elongated aggregate in a mixture resulted in a lower resistance to shear deformation [5]. Hamzah et al found that the density, stability and air void for all mixtures incorporated geometrically cubical aggregates were significantly higher than those of the other Marshall properties [6]. Janoo concluded that shape, angularity, and roughness have significant effect on base performance. He stated that several studies have shown that there can be as mush as 50 percent change in resilient modulus of base materials due to geometric irregularities of coarse and fine aggregate particles [7]. Stephens and Sinha presented data on the effect of aggregate shape, and recommended blends of regular particles, flat particles, and rod-like particles to achieve optimum strength. He concluded that effect of blend aggregate shapes on density isn't large, voids decrease with increasing asphalt; higher voids always occurring in mixes $30 \%$ or more of flat aggregate particles and increases of asphalt above $6 \%$ cause large reduction in stability unless the aggregate is a blend of shapes approaching $50 \%$ regular $-25 \%$ flat and $25 \%$ rod [8].

Aggregate gradation and surface texture have also important effect on HMA properties. Several researches performed to study the effect of aggregate gradation on the characteristics of HMA. In 1992, Kim et al evaluated the effects of aggregate gradation on permanent deformation. The analysis revealed that aggregate type has a significant effect on the fatigue resistance and permanent deformation of asphalt mixtures [3]. Oduroh et al. showed that the percentage of crushed coarse particles had a significant effect on laboratory permanent deformation properties. As the percentage of crushed coarse particles decreased, the rutting potential of the HMA mixtures increased [9]. Kandhal and Mallick found that the rutting potential for binder courses with larger aggregates is less than surface courses with finer aggregates and higher binder content [10]. Barksdale et al. had observed a similar trend in their data, and found that rutting in the surface courses was reduced by approximately $13 \%$ using a coarser HMA [11]. Bose reported that, permanent strain decreased with increased aggregate size in large stone mixes. Large size aggregate has led to lower binder content, high density, satisfying voids in mineral aggregate [12]. Krutz and Sebaaly studied effect of aggregate gradation on behavior of HMA by designing asphalt concrete mixtures to include more coarse aggregate; a mixture more resistant to rutting will be produced [13]. Monismith found that aggregate shape and surface texture characteristics have an influence on the fatigue life and stiffness characteristics of HMA. His research recommended utilizing rough-textured materials with dense gradation for thick pavements in order to increase mix stiffness and fatigue life; whereas, it might be acceptable to utilize smooth-textured aggregates in thin pavements since they produce less stiff mixtures resulting in increased fatigue life [14]. Saeed, et al. revealed that lack of adequate particle angularity and surface texture is one of the contributing factors to fatigue cracking and rutting in asphalt pavements, while lack of adequate particle angularity and surface texture is a contributing factor to cracking in concrete pavement [15]. Stakston and Bahia also have indicated that rut resistance is "highly dependent on aggregate grading, and that mixes made with the best possible materials would fail without a proper gradation [16]. In 1999, El-Basyouny and Mamlouk evaluated the effect 
of aggregate gradation on the rutting potential. They found that both the aggregate gradation and aggregate nominal size affected the rut depth for pavement section [17].

The objective of this study was to investigate the effect of the aggregate shape in hot mix asphalt (HMA). The parameters studied in this pace of paper were aggregate gradation, aggregate shape and aggregate surface texture. Marshal test and indirect tensile test were conducted to study the effect of these parameters on HMA.

\section{Materials used}

The crushed gravely soil was used as a coarse aggregate, siliceous sand as a fine aggregate and limestone powder as mineral filler. Two different sizes of coarse aggregate were used named S1 and S2. The gradation of the combined aggregate along with the specification limit is presented in Fig.1. The bulk specific gravities of coarse aggregate, fine aggregate and mineral filler used were 2.628, 2.634 and 2.652 respectively. The Los Angeles abrasion of coarse aggregate was $25.3 \%$. The asphalt cement used was AC 60/70. This asphalt material, supplied by El-Suez is the usual asphalt grade used for asphalt pavement construction in Egypt. The specific gravity of asphalt cement used was 1.03.

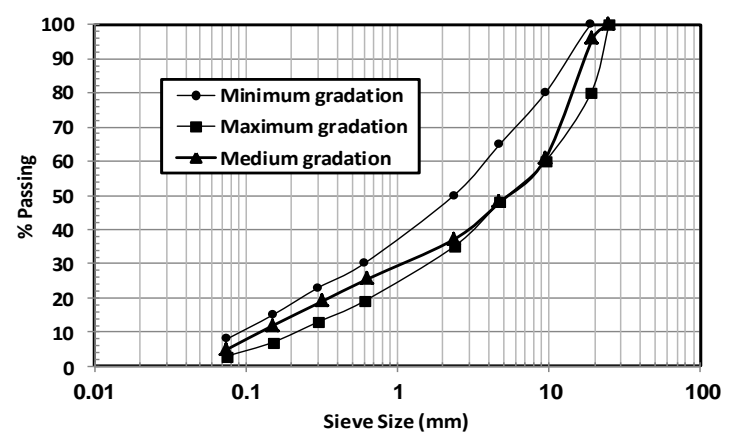

Fig. 1. Gradation curve for coarse aggregate, fine aggregate and mineral filler.

\section{Shape of aggregate determination}

The suitability of aggregates used in asphalt construction is evaluated in terms of many properties. These properties include grading, practical shape and surface texture. The shape of aggregate was determined through two methods, image measurements of coarse aggregate and the particle shape index.

\subsection{Image measurements of coarse aggregate}

Aggregate shape analysis was carried out through the use of the Zingg diagram (1935) on the basis of the particle longest diameter $(d L)$, the intermediate diameter $(d I)$, and the shortest diameter $(d S)$ The elongation ratio and the flatness ratio were used to define the aggregate shape as shown in Fig. 3 The former is the ratio of $d I$ to $d L$, and the latter is the ratio of $d S$ to $d I$. Three different aggregate shapes were selected as follows: disk, blade and cubical. The disk aggregate was selected for the HMA mix in order to contrast it with the mixes consisting of other shapes. The disk-shaped aggregate is flaky and oblate and the blade-shaped is elongated. Some of results were shown in Table 2. 


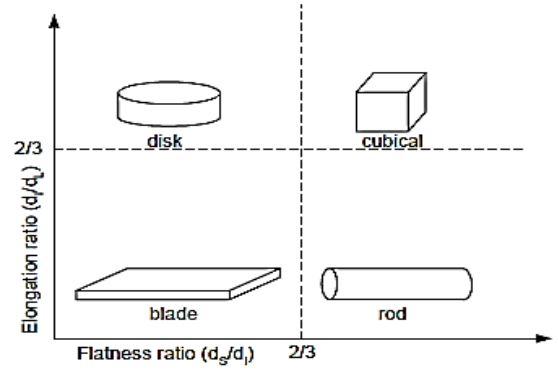

Fig. 2. Zingg diagram for aggregate shape limits.

Table 2.

estimation of the sample dimension for crushed gravel soil S2 (cubical shape).

\begin{tabular}{|c|c|c|c|c|c|c|c|c|}
\hline $\begin{array}{c}\text { sieve } \\
\text { size } \mathbf{m m}\end{array}$ & sample & $\begin{array}{c}\text { dl } \\
\text { (Long) }\end{array}$ & $\begin{array}{c}\text { dI } \\
\text { (medium) }\end{array}$ & $\begin{array}{c}\mathbf{d S} \\
\text { (short) }\end{array}$ & Elongation & flatness & shape & sphericity \\
\hline \multirow[b]{8}{*}{$25-19$} & 1 & 22.4 & 19.7 & 18.8 & 0.8794 & 0.839 & 0.894 & 0.9037 \\
\hline & 2 & 21.7 & 21.4 & 20.2 & 0.9861 & 0.930 & 0.9373 & 0.9718 \\
\hline & 3 & 25.1 & 20.5 & 18.2 & 0.8167 & 0.7251 & 0.8023 & 0.8397 \\
\hline & 4 & 23.3 & 20.8 & 18 & 0.8927 & 0.772 & 0.8176 & 0.8835 \\
\hline & 5 & 25 & 22.5 & 19 & 0.9 & 0.76 & 0.8011 & 0.8810 \\
\hline & 6 & 25.5 & 21 & 19.6 & 0.8235 & 0.768 & 0.8469 & 0.8586 \\
\hline & \multicolumn{4}{|r|}{ Average } & 0.8831 & 0.799 & 0.8500 & 0.8897 \\
\hline & \multicolumn{4}{|c|}{ Aggregate Shape } & Shape & IS & \multicolumn{2}{|l|}{ Cubical } \\
\hline \multirow[b]{8}{*}{$19-12.5$} & 1 & 17.4 & 16.1 & 154 & 0.9252 & 8.850 & 9.2009 & 2.0156 \\
\hline & 2 & 20.2 & 18.3 & 16.8 & 0.9059 & 0.831 & 0.873 & 0.9099 \\
\hline & 3 & 18.1 & 16.6 & 14.9 & 0.9171 & 0.823 & 0.8595 & 0.9105 \\
\hline & 4 & 16.7 & 16.2 & 15.5 & 0.9700 & 0.928 & 0.9423 & 0.9656 \\
\hline & 5 & 18.7 & 14.2 & 14.2 & 0.7593 & 0.759 & 0.8714 & 0.8323 \\
\hline & 6 & 18.3 & 16.7 & 14.8 & 0.9125 & 0.808 & 0.8465 & 0.9037 \\
\hline & \multicolumn{4}{|r|}{ Average } & 0.8983 & 2.166 & 2.2657 & 1.0896 \\
\hline & \multicolumn{4}{|c|}{ Aggregate Shape } & Shape & IS & \multicolumn{2}{|l|}{ Cubicle } \\
\hline \multirow[b]{8}{*}{$12.5-9.5$} & 1 & 15 & 11.7 & 10 & 0.78 & 0.666 & 0.7548 & 0.8041 \\
\hline & 2 & 13 & 10.5 & 10.1 & 0.8076 & 0.776 & 0.8644 & 0.8561 \\
\hline & 3 & 13.7 & 13.2 & 11 & 0.9635 & 0.802 & 0.8179 & 0.9179 \\
\hline & 4 & 12.8 & 12.1 & 11.1 & 0.945 & 0.867 & 0.8919 & 0.9359 \\
\hline & 5 & 12.6 & 12.3 & 9 & 0.9761 & 0.714 & 0.7229 & 0.886 \\
\hline & 6 & 14 & 12.8 & 9.5 & 0.9142 & 0.678 & 0.7096 & 0.852 \\
\hline & \multicolumn{4}{|r|}{ Average } & 0.8978 & 0.751 & 0.7936 & 0.8756 \\
\hline & & & Aggreg & Shape & Shape & Is & \multicolumn{2}{|l|}{ Cubical } \\
\hline
\end{tabular}

\subsection{Particle index of coarse aggregate}

The combined effects of particle shape and surface texture of an aggregate were determined in accordance with ASTM Test Method for Index of Aggregate Particle Shape and Texture (D 3398). The equipment required for this test consists basically of a cylindrical steel mold $152 \mathrm{~mm}$ in diameter by $178 \mathrm{~mm}$ high, and steel rod $16 \mathrm{~mm}$ in diameter by $610 \mathrm{~mm}$ long with the tamping end rounded to a hemispherical tip. A clean, washed, oven-dried, single-size aggregate fraction was used for this test. The mold was filled in three equal layers, with each layer compacted with 10 well-distributed blows of the tamping rod. Each tamp consisted of a drop with the tamping rod from $51 \mathrm{~mm}$ above 
JES, Assiut University, Faculty of Engineering, Vol. 42, No. 6, November 2014, pp. 1349 - 1366

the surface of the layer being compacted. This procedure was repeated using the same material but applying 50 blows on each of the three layers. The weight of the contents of the mold in each case was determined and the corresponding percentage of voids was calculated using the bulk specific gravity of each aggregate fraction. Some of results were shown in Tables 2 . The particle index $(P I)$ is derived using the following equation:

$$
P I=1.25 V 10-0.25 V 50-32
$$

Where

$V 10=$ percent voids in the aggregate compacted with 10 blows per layer;

$V 50=$ percent voids in the aggregate compacted with 50 blows per layer.

Elongation ratio $=\frac{d_{I}}{d_{L}}$

Flatness ratio $=\frac{\mathrm{d}_{\mathrm{S}}}{\mathrm{d}_{\mathrm{L}}}$

Shape Factor $=\frac{\mathrm{d}_{\mathrm{S}}}{\sqrt{d_{I} \times d_{L}}}$

Sphericity $=\sqrt[3]{d_{\mathrm{S}} \times\left(\frac{\mathrm{d}_{\mathrm{I}}}{\mathrm{d}_{\mathrm{L}}^{2}}\right)}$

Table 2.

Estimation weighted value of particle index for crushed gravel example

\begin{tabular}{|c|c|c|c|}
\hline Sieve size & \% Retained & $\mathbf{P I}$ & Weighted value \\
\hline $25-19$ & 6.7 & 10.5945 & 0.70982882 \\
\hline $19-12.5$ & 41.2 & 11.4113 & 4.7014556 \\
\hline $12.5-9.5$ & 31.5 & 12.7184 & 4.006296 \\
\hline \multirow{3}{*}{$9.5-4.75$} & 20.6 & 12.807 & 2.638242 \\
\hline & & & 12.05582242 \\
\hline & \multicolumn{2}{|l|}{ aggregate shape } & blade \\
\hline $25-19$ & 6.7 & 11.8871 & 0.7964357 \\
\hline $19-12.5$ & 41.2 & 12.9839 & 5.3493668 \\
\hline $12.5-9.5$ & 31.5 & 13.1343 & 4.1373045 \\
\hline \multirow[t]{2}{*}{$9.5-4.75$} & 20.6 & 13.2179 & 2.7228874 \\
\hline & & & 13.0059944 \\
\hline \multicolumn{2}{|r|}{ aggregate shape } & \multicolumn{2}{|l|}{ is disk } \\
\hline $25-19$ & 6.7 & 13.0717 & 0.87580122 \\
\hline $19-12.5$ & 41.2 & 14.1246 & 5.8193352 \\
\hline $12.5-9.5$ & 31.5 & 13.8321 & 4.35711465 \\
\hline \multirow[t]{2}{*}{$9.5-4.75$} & 20.6 & 14.6511 & 3.01811836 \\
\hline & & & 14.07036943 \\
\hline \multicolumn{2}{|r|}{ aggregate shape } & is & Cubical \\
\hline
\end{tabular}




\section{Experimental work}

The coarse aggregate, fine aggregate, mineral filler and asphalt cement were used to prepare the Marshal Test specimens with $65 \mathrm{~mm}$ height and $101.6 \mathrm{~mm}$ diameter. The tested asphaltic concrete mixes were composed of $60 \%$ coarse aggregate, $36 \%$ fine aggregate, $4 \%$ mineral filler and asphalt cement. Five different asphalt contents (4, 4.5, 5, 6 and 7\%) were used to prepare Marshal Test specimens. Specimens were compacted with 50 blows on each side. Three samples were made for each asphalt content. The parameters studied in this research were aggregate shape, aggregate gradation and aggregate surface texture. The results of Marshal Design indicated that the optimum asphalt content for control mixtures was $5.3 \%$. Marshal test and indirect tensile test were conducted to study these parameters.

\subsection{Marshal test}

Marshal Stability and flow were determined using Marshal Stability apparatus at $60 \mathrm{C}^{\circ}$ of sample test. Bulk density and void ratio were determined. Marshal quotient (MQ) is an indicator of the resistance against the deformation of the asphalt concrete. MQ values are calculated to evaluate the resistance of the deformation of the all mixtures. The Marshall quotient (MQ) was determined using the following equation:

$$
\mathrm{MQ}=\frac{\text { Stability }}{\text { Flow }} \quad \mathrm{N} / \mathrm{mm}
$$

\subsection{Indirect tensile test}

The stiffness modulus and the indirect tensile strength of HMA are used often to evaluate the relative quality of materials. The load was applied vertically in the vertical diameter plane of a cylindrical specimen of asphalt concrete through a curved loading strip. The difference between this test and Marshall Test is only in the test mould. Marshall Test specimens were also used for this test having a diameter of $100 \mathrm{~mm}$ and a height from 60 to $65 \mathrm{~mm}$. The measured load value at failure $(\mathrm{P})$ in Newton, the thickness of specimen (h) in $\mathrm{mm}$ and the diameter of specimen (d) in $\mathrm{mm}$, were used to calculate the Indirect Tensile Strength Values (St) by using the following equation:

$$
\text { ITS }=\frac{2 P}{\pi \times h \times d} \quad N / \mathrm{mm}^{2}
$$

The measured lateral deformation (diagonal) $(\mathrm{U})$ and the diameter of specimen (d) were used to calculate the strain at failure $\left(\xi_{\mathrm{f}}\right)$ using the following equation:

$$
\zeta_{f}=\frac{6.63 U}{\pi d} \%
$$

The measured load at failure (P) in Newton, the thickness of specimen (h) in mm, and the lateral deformation (diagonal) value $(\mathrm{U})$ in $(\mathrm{mm})$ were used to calculate the Stiffness Modulus $\left(\mathrm{S}_{\mathrm{M}}\right)$ using the following equation:

$$
S_{M}=\frac{0.573 P}{h \times U} \quad\left[\mathrm{~N} / \mathrm{mm}^{2}\right]
$$




\section{Marshall test results and analysis}

\subsection{Effect of aggregate shape}

\subsubsection{Effect of aggregate shape on marshal stability}

Figure 3 shows the test results of Marshal Stability of aggregate shape (dish, blade and cubical).It reveals that the stability values increased with used cubical aggregate shape than disk and blade shape respectively. This result may come up because cubical aggregate shape aggregate had overlap and a strong correlation between the particles which made the mix had the best performance in resisting loads. The blade aggregate shape had the lowest value of stability this result may come up because the blade shape is a weak shape which leads to weak mix.

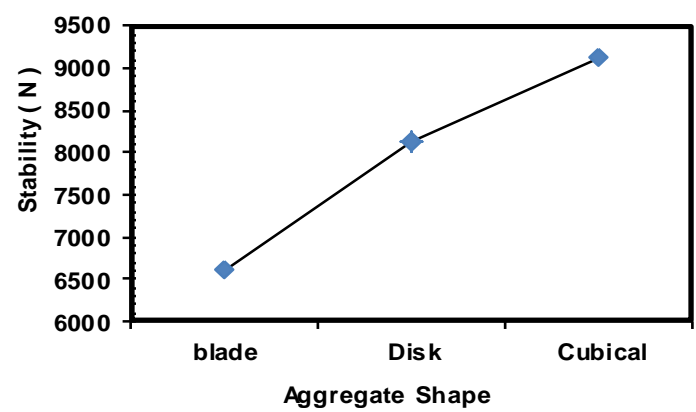

Fig. 3. Effect of aggregate shape on Marshal Stability

\subsubsection{Effect of aggregate shape on flow values}

Figure 4 shows the test results of Marshal Flow for all shape of aggregate (disk, blade and cubical). It is clear from the result that disk aggregate shape had the highest value of flow. This may come up because the disk aggregate mix had high values of voids and low density so the mix not able to resist deformation. The cubical shape had the lowest value of flow this result may come up because the mix had high density and low voids so the mix able to resist deformation.

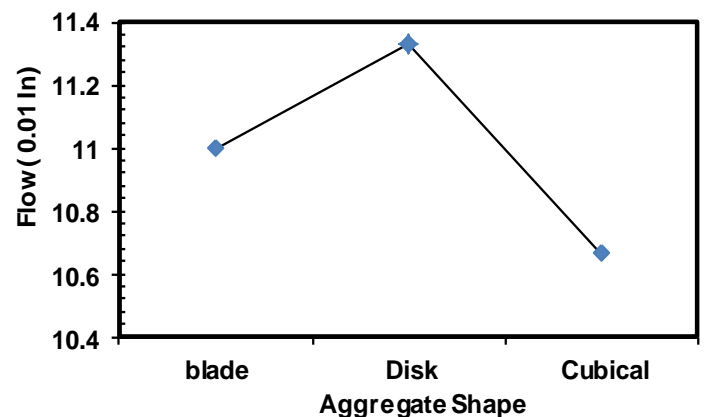

Fig. 4. Effect of aggregate shape on Flow

\subsubsection{Effect of aggregate shape on bulk density}

The relationships between aggregate shape and Marshal Bulk density are illustrated in Figure 5. It is clear from the results that the cubical and blade aggregate shape had the highest value of bulk density (slightly difference) that may come up because its good workability which let the specimen to compact well and the mix had overlap and a strong correlation between the grains. The disk aggregate shape had the lowest value of density because it had more voids than others. 


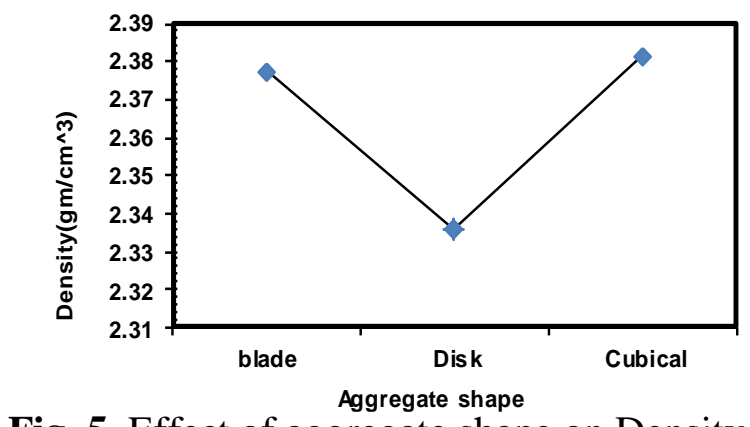

Fig. 5. Effect of aggregate shape on Density

\subsubsection{Effect of aggregate shape on air voids}

Figure 6 shows air voids values (VTM) versus aggregate shape. It reveals that the cubical and blade aggregate shape had the lowest value of air voids (slightly difference). This may come up because it's good compaction as shown from its high bulk density while the disk aggregate shape had the highest value of VTM that may be because the disk aggregate shape had low value of density.

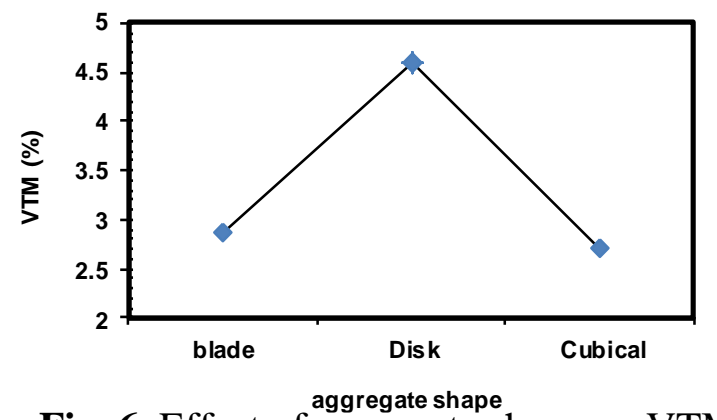

Fig. 6. Effect of aggregate shape on VTM

\subsubsection{Effect of aggregate shape on Marshall Quotient}

The relationships between aggregate shape and Marshal Quotient (MQ) are illustrated in figure 7. It reveals that the cubical aggregate shape had the highest value of MQ than disk and blade shapes respectively. This result may come up because the cubical aggregate shape had high value of stability and low flow value. The blade aggregate shape had the lowest value of MQ that may come up because it had the high value of flow and low stability flow.

\subsection{Effect of surface texture of aggregate}

\subsubsection{Effect of surface texture of aggregate on marshal stability}

Figure 8 shows the test results of stability of all type of aggregate surface texture (Smooth and Rough). It reveals that the stability increased with used rough surface texture aggregate particles than smooth surface texture aggregate. This result may come up because aggregate particles with rough surface texture given strong homogenous mix enough to resist loads than smooth particles.

\subsubsection{Effect of aggregate surface texture on flow values.}

Figure 9 shows the test results of flow for all type of aggregate surface texture (smooth and rough surface texture).It reveals that the flow value increased with used smooth aggregate particles. It is clear from the result that smooth aggregate particles mix had the highest value of flow. This may come up because rough particles are difficult to compact. It may be contain some of voids which made the mix not able to resist flow. 


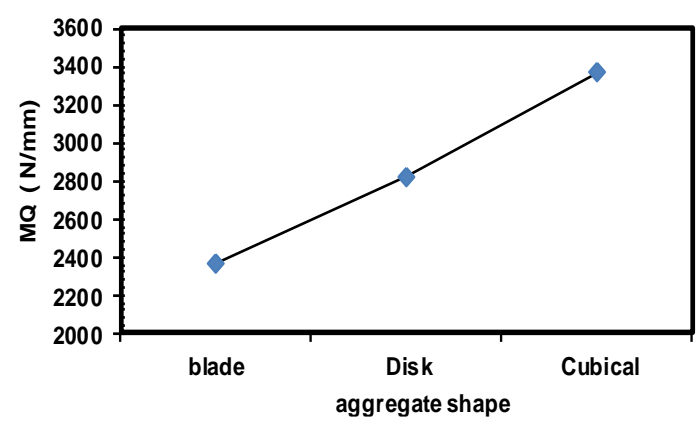

Fig. 7. Effect of aggregate shape on Marshal Quotient (MQ).

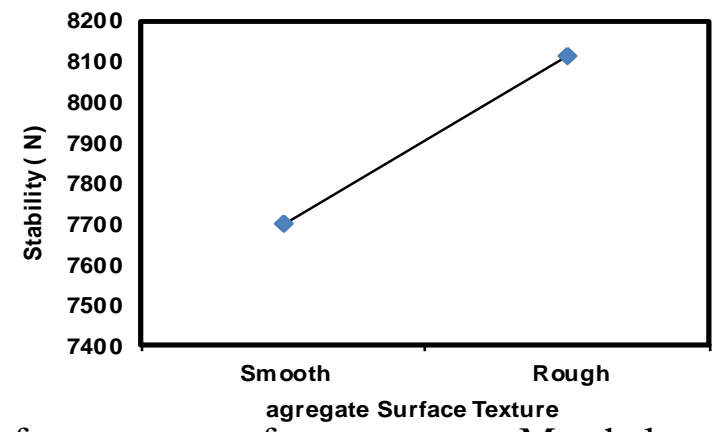

Fig. 8. Effect of aggregate surface texture on Marshal surface texture.

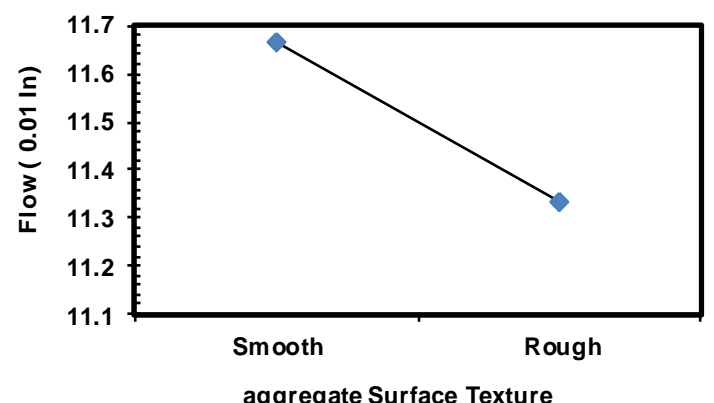

Fig. 9. Effect of aggregate surface texture on flow

\subsubsection{Effect of aggregate surface texture on bulk density}

The relationships between aggregate surface texture and bulk density are illustrated in Figure 10.It is clear from the results that rough particles had the highest value of bulk density than smooth particles. That may come up because its good workability which let the specimen to compacted well than rough aggregate particles.

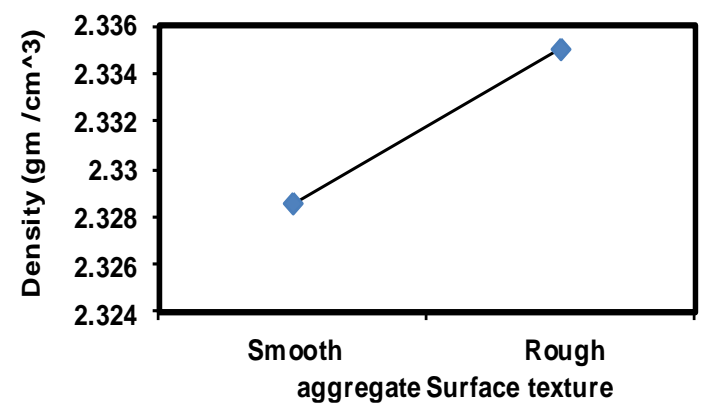

Fig. 10. Effect of aggregate surface texture on Density 


\subsubsection{Effect of aggregate surface texture on air voids}

Figure 11 shows air voids values (VTM) versus aggregate surface texture .It reveals that the rough aggregate particles had the lowest value of air voids that may be because it's good compaction as shown from its high bulk density than smooth particles which were difficult to compact, so it had the lowest value of bulk density.

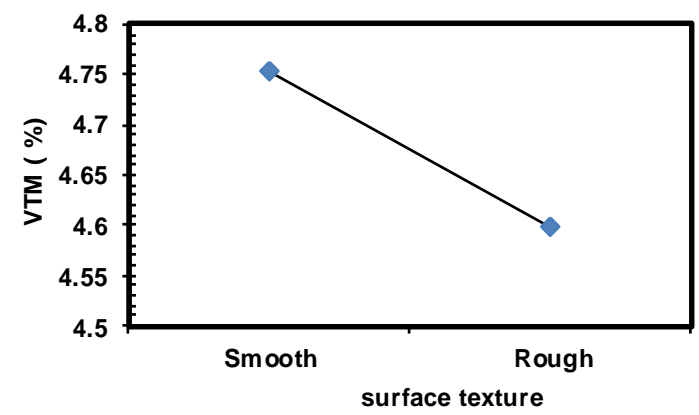

Fig. 11..Effect of aggregate surface texture on VTM.

\subsubsection{Effect of aggregate surface texture on Marshall Quotient}

The relationships between aggregate surface texture and Marshal Quotient (MQ) are illustrated in figure 12. It reveals that smooth aggregate particles had the lowest value of MQ than smooth particles. This result may come up because smooth particles had high flow and thus it had low resistance against the deformation of the asphalt concrete, while rough particles had the highest value of MQ as it had low flow which mad the mix had high capility to resist deformation.

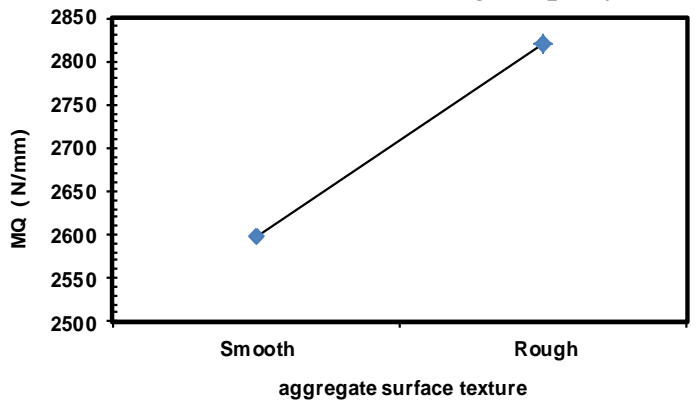

Fi g.12..Effect of aggregate surface texture on MQ

\subsection{Effect of aggregate gradation}

\subsubsection{Effect of aggregate gradation on marshal stability}

Figure 13 shows the test results of stability of aggregate gradation (maximum, medium and minimum). It reveals that the stability increased with used medium aggregate gradation than maximum and minimum gradation respectively. This result may come up because medium aggregate particles contain good percentage of aggregate particle size ( $\mathrm{S} 1$ and S2) in mix which made the mix had the best performance in resisting loads.

\subsubsection{Effect of aggregate gradation on flow values}

Figure 14 shows the test results of flow for all type of aggregate gradation (maximum, medium and minimum). It is clear from the result that minimum aggregate gradation had the highest value of flow than medium and maximum aggregate gradation. This may come up because the minimum aggregate gradation contain large amount of small particle (S1) with compare to S2 which made the mix weak to resist the strain. The medium and maximum aggregate gradation has nearly the same flow values. 


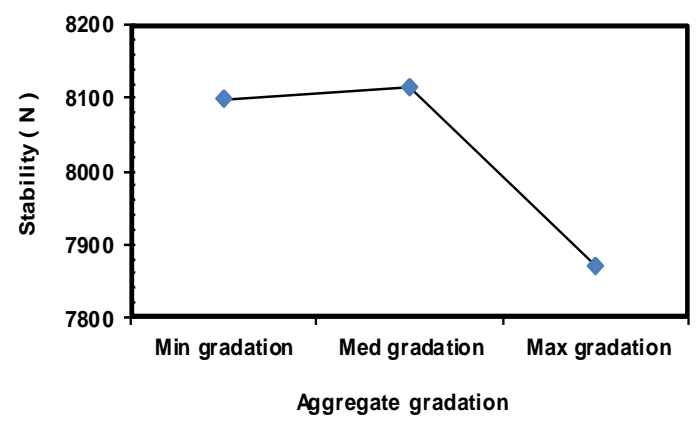

Fig. 13..Effect of aggregate gradation on Marshal Stability

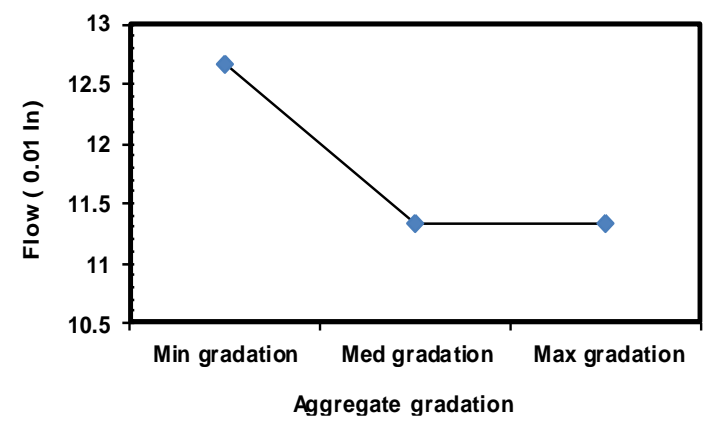

Fig. 14. Effect of aggregate gradation on Flow

\subsubsection{Effect of aggregate gradation on bulk density}

The relationships between aggregate gradation and bulk density are illustrated in Figure 15. It is clear from the results that maximum aggregate gradation had the highest value of bulk density that may come up because its good workability which let the specimen to compact well. The medium aggregate gradation had the lowest value of density because it had more voids than the others.

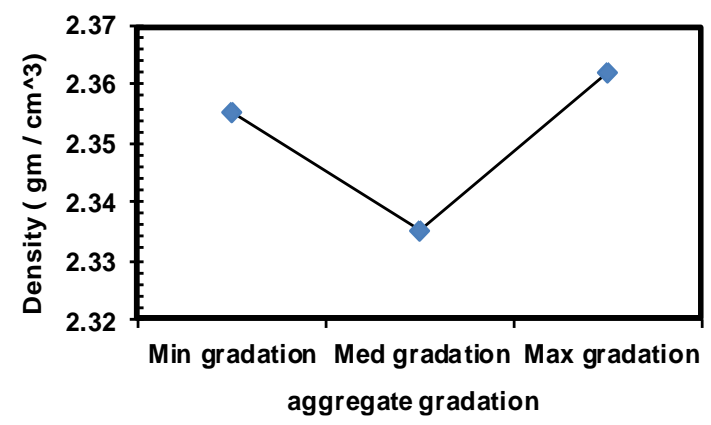

Fig. 15. Effect of aggregate gradation on Density

\subsubsection{Effect of aggregate gradation on air voids}

Figure 16 shows air voids versus aggregate gradation .It reveals that medium aggregate gradation had the highest value of air voids than minimum and medium gradation respectively. This may be because it's poor compaction as shown from its low bulk density while the maximum gradation had the lowest value of VTM and this may come up because it had the highest value of bulk density. 


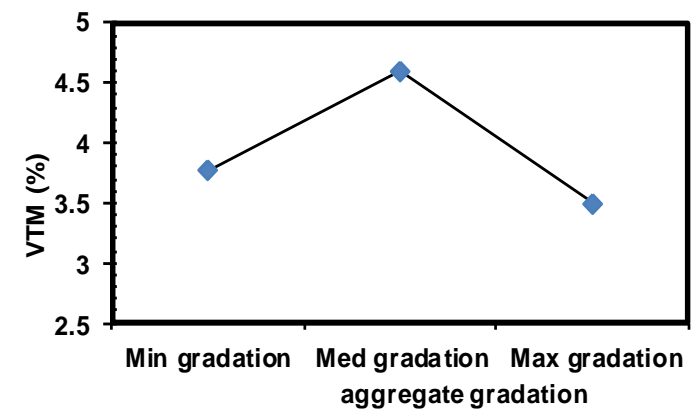

Fig. 16. Effect of aggregate gradation on VTM

\subsubsection{Effect of aggregate gradation on Marshall Quotient}

The relationships between aggregate gradation and Marshal Quotient (MQ) are illustrated in figure 17. It reveals that medium aggregate gradation had the highest value of MQ than maximum and minimum aggregate particle. This result may come up because medium aggregate gradation had high value of stability and low flow value. The minimum aggregate gradation had the lowest value of MQ that may come up because it had the high value of flow and low stability flow.

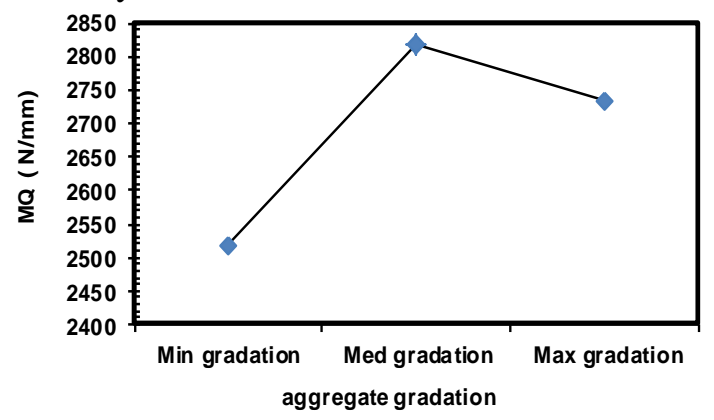

Fig. 17. Effect of aggregate gradation on MQ

\section{Indirect tensile test results}

\subsection{Effect of shape of aggregate}

\subsubsection{Effect of shape of aggregate on indirect tensile strength}

Figure 18 shows the test results of indirect tensile strength (ITS) for all shapes of aggregate (disk, blade and cubical shape).It reveals that the indirect tensile strength value increased with used crushed gravel mix with cubical particles shape than disk and blade shape respectively. This result may come up because Cubical aggregate shape had high interlock and internal friction between aggregate particles which lead the mix had good internal resistance against external loads as it noted from its high value of the loss Angelus test and Marshal Stability. Blade aggregate shape had the lowest value of ITS. This may come up because its weak internal resistance according to its weak interlock between particles as it noted from its low value of Marshal Stability.

\subsubsection{Effect of shape of aggregate on stiffness modulus}

Figure 19 shows the test results of stiffness modulus $\left(\mathrm{S}_{\mathrm{M}}\right)$ for all shapes of aggregate (disk, blade and cubical shape).It reveals that the stiffness modulus value increased with used crushed gravel mix with cubical particles shape than disk and blade shape 
respectively. This result may come up because cubical aggregate shape had high interlock and internal friction between aggregate particles which lead the mix had good internal resistance against external loads as it noted from its high value of the loss Angelus test and Marshal Stability. Blade aggregate shape had the lowest value of $S_{M}$. This may come up because its weak internal resistance according to its weak interlock between particles as it noted from its low value of Marshal Stability.

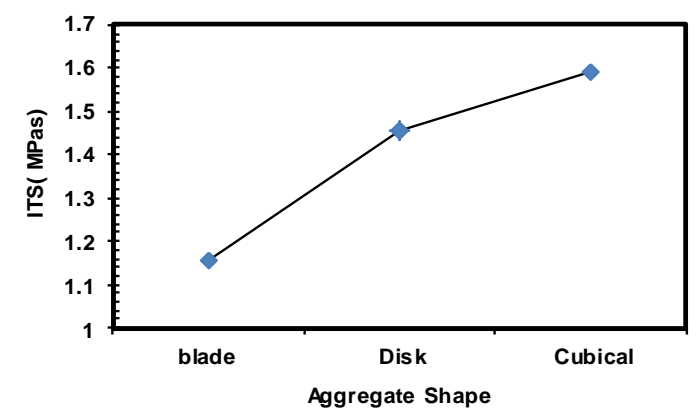

Fig. 18. Effect of shape of aggregate on ITS

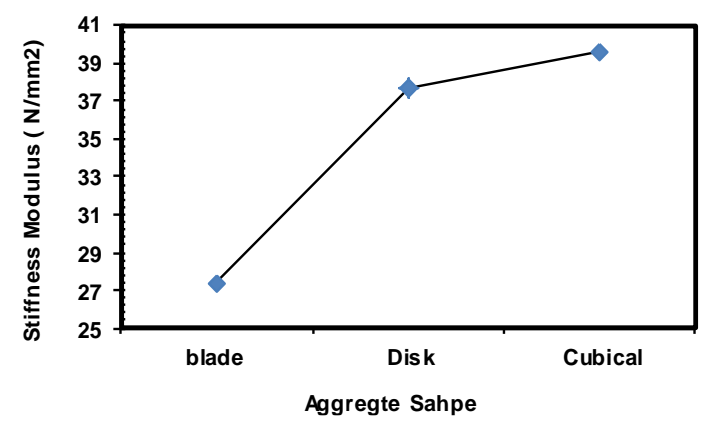

Fig. 19. Effect of shape of aggregate on Stiffness Modulus.

\subsubsection{Effect of shape of aggregate on failure strain}

Figure 20 shows the test results of failure strain for all shapes of aggregate (disk, blade and cubical shape). It is clear from results that blade aggregate particle shape mix had the highest value of failure strain than disk and cubical particles shape respectively. This may come up because it had low internal resistance against horizontal deformation as noted from its low value of ITS. Cubical particle shape mix had the lowest value of failure strain this maybe because it had high internal resistance against loads and horizontal deformation as it noted from its high value of ITS.

\section{2. Effect of surface texture of aggregate}

\subsubsection{Effect of surface texture of aggregate on indirect tensile strength}

Figure 21 shows the test results of indirect tensile strength (ITS) for all surface texture of aggregate (smooth and rough texture).It reveals that the indirect tensile strength value increased with used crushed gravel mix with rough surface texture than smooth texture. This result may come up because rough aggregate particle had high interlock and internal friction between aggregate particles which lead the mix had a good internal resistance against external loads as it noted from the loss Angelus test and Marshal Stability value. Smooth aggregate particle had the lowest value of ITS. 
This may come up because its weak internal resistance according to its weak interlocks between particles as shown form its low value of stability.

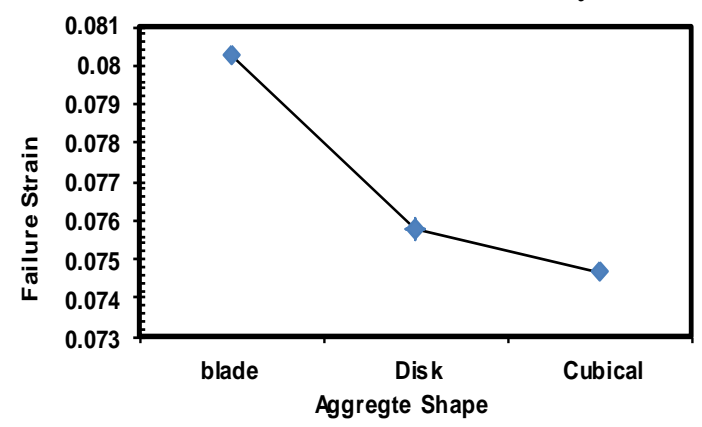

Fig. 20. Effect of shape of aggregate on Failure strain.

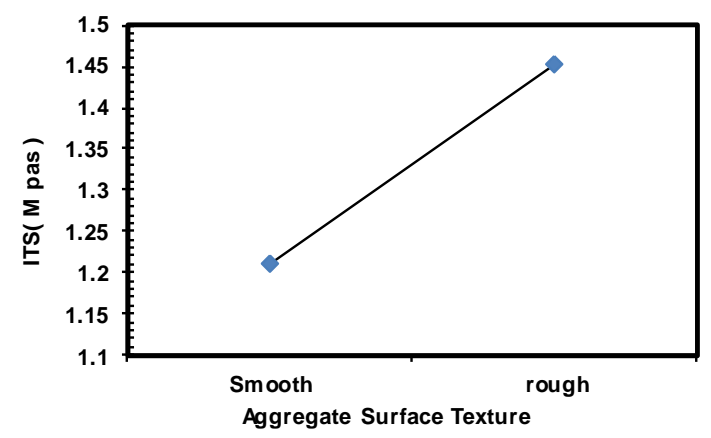

Fig. 21. Effect of surface texture of aggregate on ITS

\subsubsection{Effect of surface texture of aggregate on stiffness modulus}

Figure 22 shows the test results of stiffness modulus $\left(S_{M}\right)$ for all surface texture of aggregate (smooth and rough texture). It reveals that the stiffness modulus value increased with used crushed gravel mix with rough surface texture than smooth texture. This result may come up because rough aggregate particle had high interlock and internal friction between aggregate particles had good internal resistance against external loads as is evident from its high value of ITS. Smooth aggregate particle had the lowest value of stiffness modulus $\left(\mathrm{S}_{\mathrm{M}}\right)$ this may come up because its weak internal resistance according to its weak interlock between particles as shown form its low value of ITS.

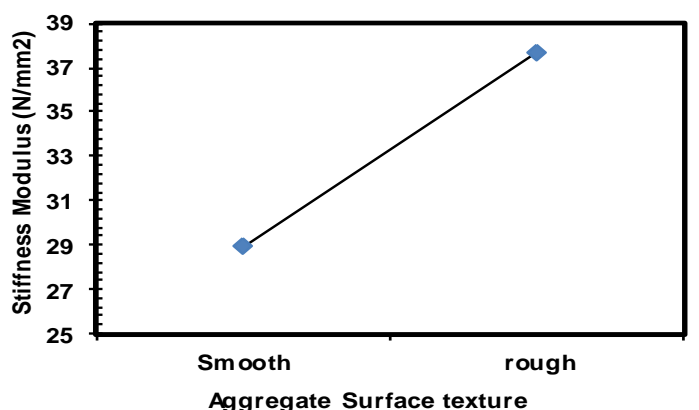

Fig. 22. Effect of surface texture of aggregate on stiffness modulus

\subsubsection{Effect of surface texture of aggregate on failure strain}

Figure 23 shows the test results of failure strain for all surface texture of aggregate (smooth and rough texture). It is clear from results that smooth particles mix had the 
highest value of failure strain than rough particles. This may come up because it had low internal resistance against external loads which made the specimen had low resistance against horizontal deformation as it noted from its low value of ITS.

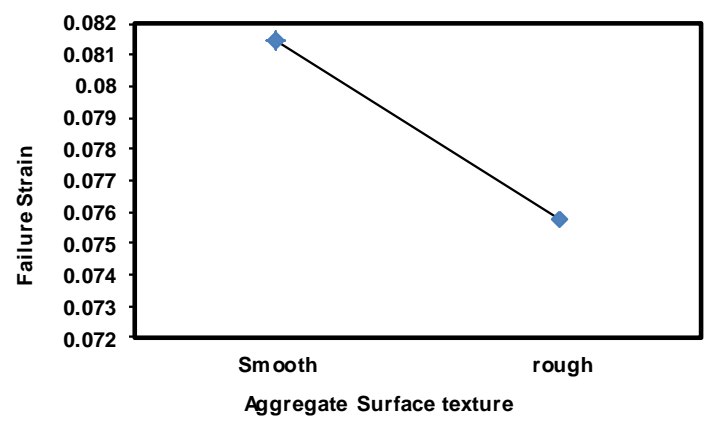

Fig. 23. Effect of surface texture of aggregate on Failure Strain

\subsection{Effect of gradation of aggregate}

\subsubsection{Effect of gradation of aggregate on indirect tensile strength}

Figure 24 shows the test results of indirect tensile strength (ITS) for all types of aggregate gradation (maximum, medium and minimum gradation). It reveals that the indirect tensile strength value increased with used medium aggregate gradation for crushed gravel mix than max. and min. gradation respectively. This result may come up because medium gradation had a good percentage of aggregate particles size (S1and S2) which made the specimen aggregate particles had good internal resistance against external loads as it noted from its high value of Marshal Stability. Maximum Aggregate gradation had the lowest value of ITS. This may come up because its weak internal resistance according to its weak interlocks between particles as shown form its low value of stability.

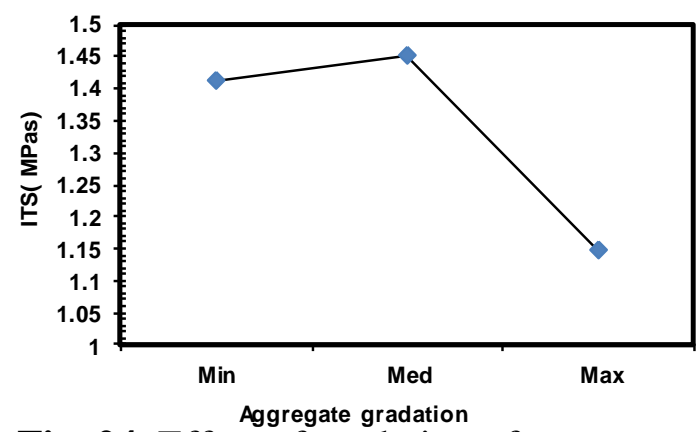

Fig. 24. Effect of gradation of aggregate on ITS

\subsubsection{Effect of gradation of aggregate on stiffness modulus}

Figure 25 shows the test results of stiffness modulus $\left(S_{M}\right)$ for all types of aggregate gradation (maximum, medium and minimum gradation).It reveals that the stiffness modulus value increased with used medium aggregate gradation for crushed gravel mix than max. and min. gradation respectively. This result may come up because medium gradation had good internal resistance against external loads as it noted from it high value of ITS. Maximum aggregate gradation had the lowest value of stiffness modulus $\left(\mathrm{S}_{\mathrm{M}}\right)$ this may come up because its weak internal resistance to external loads as it noted from its low value of ITS. 


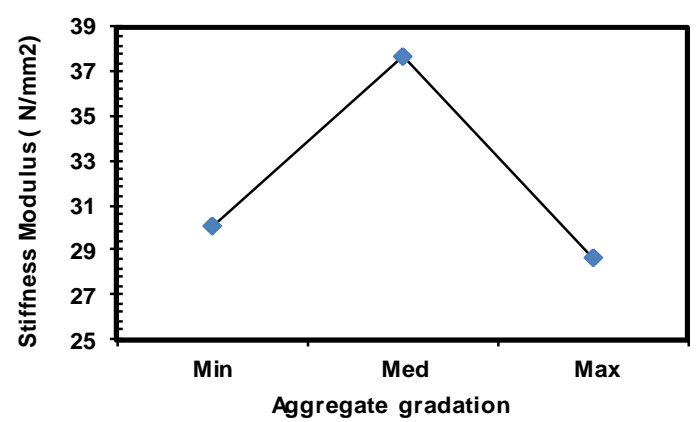

Fig. 25. Effect of gradation of aggregate on Stiffness Modulus.

\subsubsection{Effect of gradation of aggregate on failure strain}

Figure 26 shows the test results of failure strain for all gradations of aggregate (maximum, medium and minimum gradation).It reveals that the failure strain value increased with used mix with minimum aggregate gradation than med. and max gradation respectively. This may come up because samples with min. aggregate gradation had low internal resistance against as horizontal deformation as noted from its low value of Marshal Flow. Medium aggregate gradation had the lowest value of failure strain this may be because it had high internal resistance against loads and horizontal deformation as it noted from its high value of ITS and stability.

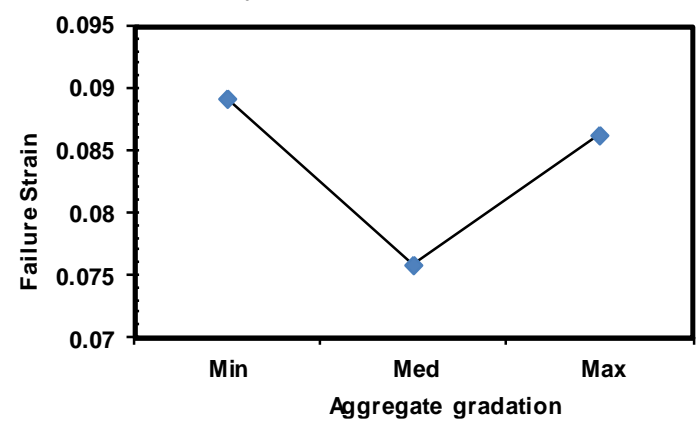

Fig. 26. Effect of gradation of aggregate on Failure strain

\section{Conclusions}

Based upon the analysis of the experimental investigation, the following conclusions are drawn:

1- Marshall Stability, bulk density, MQ were increased by using rough crushed gravel than using smooth crushed gravel. Otherwise, the values of flow and VTM were decreased by using rough particles than using smooth particles.

2- The values of stability, VTM, MQ were higher by using medium gradations comparing with max. and min. gradations. Otherwise, the values of density and flow were decreased.

3- The values of stability, density, MQ were higher by using cubical aggregate shape comparing with disk and blade shape respectively. Otherwise, the values of VTM and flow were decreased.

4- By using rough particles the values of ITS and stiffness modulus were higher than using smooth particles. Otherwise, the failure strain was lower.

5- By using medium grading the values of IT'S and stiffness modulus were higher than using min and max grading. Otherwise, the failure strain was lower. 
6- Using cubical aggregate shape increased the values of ITS and stiffness modulus than using blade and disk shape and decrease failure strain.

\section{REFERENCES}

[1] Brown, E.R., McRae, J.L., and Crawley, A.B. ,"Effect of aggregate on performance of bituminous concrete", ASTM STP 1016, Philadelphia, PP.34-63, 1989.

[2] Kandhal, P.S., Khatri, M.A., and Motter, J.B., "Evaluation of particle shape and texture Of mineral aggregates and their blends", Journal of Association of Asphalt Paving Technologists, No.61, PP. 217-240, 1992.

[3] Kim, Y. R., Yim, N., and Khosla, N. P., "Effects of Aggregate Type and Gradation on Fatigue and Permanent Deformation of Asphalt Concrete", American Society for Testing and Materials, STP 1147, West Conshohocken, Philadelphia, 1992.

[4] Kandhal, P. S., and Parker, F. J., "Aggregate Tests Related to Asphalt Concrete Performance in Pavements.", National Cooperative Highway Research Program Report 405, Transportation Research Board, National Research Council, Washington, D.C., 1998.

[5] Chen J. S, Chang M.K and LIN K.Y., "Influence of Coarse Aggregate Shape on The Strength of Asphalt Concrete Mixtures", Journal of the Eastern Asia Society for Transportation Studies, Vol. 6, PP. 1062 - 1075, 2005.

[6] Hamzah O.M, Puzi M. A. and Azizli K.A., "Properties of Geometrically Cubical Aggregate and Its Mixture Design", 2010.

[7] Janoo, V. C. "Quantification of Shape, Angularity, and Surface Texture of Base course Materials”, U.S. Army Corps of Engineers Special Report 98-1, Cold Regions Research \& Engineering Laboratory, Hanover, NH, 1998.

[8] Stephens, J.E., and Sinha, K.C. "Influence of aggregate shape on bituminous mix character", Journal of Association of Asphalt Paving Technologists, Vol.47, P 434-456, 1974.

[9] Oduroh, P. K., Mahboub, K. C., and Anderson, R. M., "Flat and elongated aggregates in Super pave regime", Journal of Materials in Civil Engineering, ASCE, 2000, Vol.12, pp.124-130.

[10] Kandhal, P. Mallick, R, "Effect of Mix Gradation on Rutting Potential of Dense-Graded Asphalt Mixtures", Washington: Transportation Research Board, 2007.

[11] Barksdale, R. D., Pollard C. O., Siegel, T., and Moeller, S., "Evaluation of the Effect of Aggregate On Rutting and Fatigue of Asphalt.", Technical Report FHWA-AG-92- 8812. Georgia Department of Transportation, Atlanta, GA, 1992.

[12] Bose S., "Development of design procedure for bituminous macadam mixes for roads and highways", Highways Res Bull, No.65, PP 93-102, 2001.

[13] Krutz, N.C., and Sebaaly, P.E., "Effect of aggregate gradation on permanent deformation of asphaltic concrete", Proceedings of Association of Asphalt Paving Technologists, No. 62, PP450-473, 1993.

[14] Monismith, C. L., "Influence of Shape, Size, and Surface Texture on the Stiffness and Fatigue Response of Asphalt Mixtures", Highway Research Board 109 Special Reports, Transportation Research Board, National Research Council, Washington, D.C., PP 4-11, 1970.

[15] Saeed, A., Hall, J., and Barker, W. "Performance-Related Tests of Aggregates for Use in Unbound Pavement Layers.” National Cooperative Highway Research Program Report 453, Transportation Research Board, National Research Council, Washington, D.C 2001).

[16] Stakston, A.D., and Bahia, H. ," The Effect of Fine Aggregate Angularity, Asphalt Content and Performance Graded Asphalts on Hot Mix Asphalt Performance", University of Wisconsin Madison, Department of Civil and Environmental Engineering, Submitted to Wisconsin Department of Transportation, Division of Transportation Infrastructure Development, Research Coordination Section, WisDOT Highway Research Study 0092-45-98, 2003.

[17] El-Basyouny, M.M. and Mamlouk, M.S, "Effect of aggregate gradation on rutting potential of Superpave mixes", Paper presented at 78th annual meeting of the Transportation Research Board, Washington D.C, 1999. 


\section{دراسة تأثير شكل حبيبات الركام على خواص الخلطات الأسفلتيه}

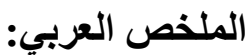

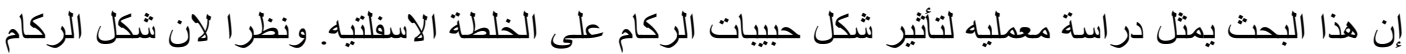

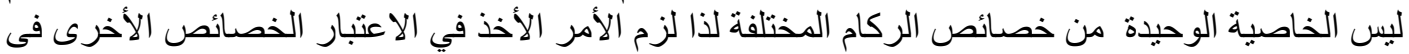

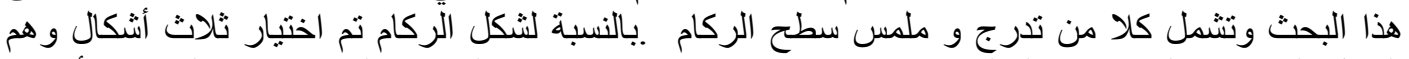

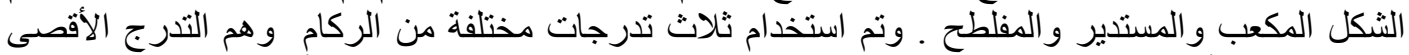

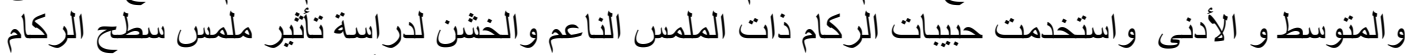

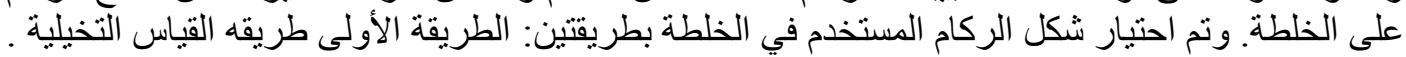

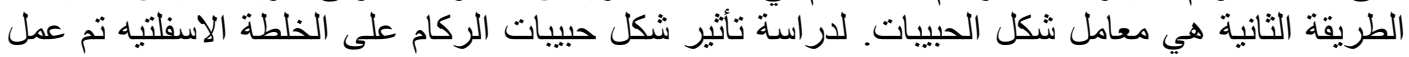

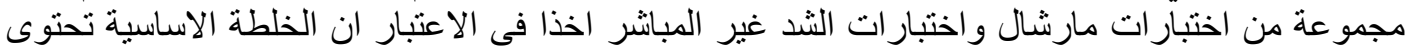

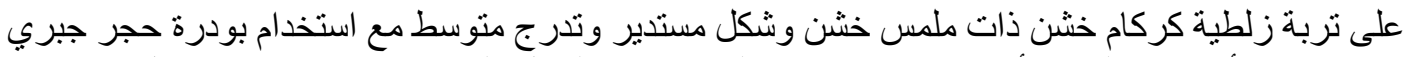

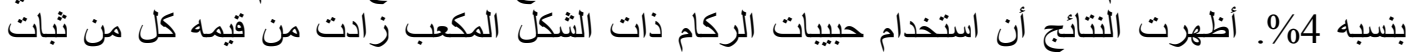

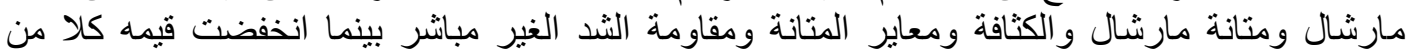

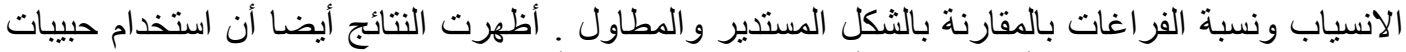

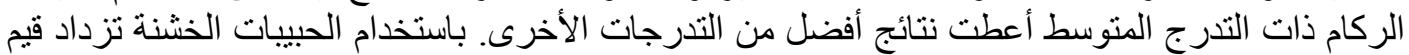

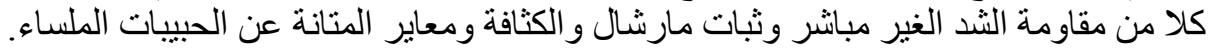

